\title{
GEOGRAFÍA MATERIAL Y SIMBÓLICA EN LOS CONCURSOS DE ARQUITECTURA EN LAS DÉCADAS DEL 60 Y 70 EN ARGENTINA: ENTRE LA EUFORIA UTÓPICA Y LAS PRÁCTICAS DEL LUGAR. MAPEO DE CASOS.
}

\section{GERVASIO ANDRÉS MEINARDY}

Universidad Nacional del Litoral - Facultad de Arquitectura, Diseño y Urbanismo. Ciudad Universitaria. S3000. Santa Fe. +54 3424575100 https://orcid.org/0000-0002-6153-0297

gmeinardy@gmail.com

\section{RESUMEN}

El presente artículo analiza la relación entre el desarrollismo -como ideario socio-económico-político- y ciertas obras y proyectos emblemáticos de la arquitectura argentina del período desarrollados mediante el instrumento del concurso. Es en este período temporal donde el sistema de concurso abierto y plural se multiplica, constituyéndose en una herramienta central en la organización, producción y transformación de la arquitectura. Para demostrar esta propuesta, realizaremos un mapeo de casos, lo que presupone indagar acerca de ciertas prácticas materiales y simbólicas, exponiendo la producción de la cultura arquitectónica local, investigando sus articulaciones más internas; de este modo revelando códigos, fuerzas y procesos que actúan en la construcción de dichas formas culturales. En contraposición al método de contratación directa de proyectos y obras, el sistema de concursos ha potenciado la implementación de ensayos, teorías, nuevas lógicas y experimentación proyectual, abriendo nuevos espacios de discusión, reflexión y articulación de manera simultánea en el seno del campo disciplinar.

PALABRAS CLAVE: Desarrollismo. Concursos.

Proyectos. Experimentación. Argentina.
Submissão: 30/12/2020

Aceite:26/04/2021

\section{ABSTRACT}

This article analyzes the relationship between developmentalism -as a socio-economic-political ideologyand specific emblematic works and designs developed through the architectural competitions in Argentine architecture between 1960s and 1970s. The open and plural contest system multiplies within the period, becoming a central tool in the organization, production, and architecture transformation. To illustrate the proposal, we will fulfill a case mapping, inquiring about particular material and symbolic practices, revealing the production of local architectural culture, exploring its internal articulations, thus revealing codes, forces, and processes that fostered cultural settings. Contrary to the direct contracting of designs and works, the contest system has promoted essays, theories, new logics, and design experimentation opening new spaces for discussion, reflection, and articulation simultaneously within the field discipline.

KEYWORDS: Developmentalism. Contests. Designs. Experimentation. Argentina. 


\section{INTRODUCCIÓN}

Las décadas de 1960 y principios de los años 1970 se reconoce como el período en el que el mecanismo de los concursos de arquitectura se difundió como práctica proyectual, abierta y plural. En este sentido, Silvestri afirma que estos se trataron "[...] de una cuestión clave en la conformación del campo arquitectónico de los años sesenta - setenta, cuando la movilidad social y la transformación del ideario dentro de la disciplina se producian [...]" (SILVESTRI, 2011, p.80), mediante la multiplicidad de esta práctica, transparente en su mecanismo y abierto a nuevos escenarios. A través del análisis de los objetos arquitectónicos se puede desentrañar la implementación de ciertas estrategias proyectuales, lo que permite inferir determinadas articulaciones y relaciones: el equipo ganador estaba constituido con frecuencia por profesionales muy jóvenes, a veces sin la suficiente experiencia, y que imponían nuevas tendencias; las vinculaciones entre el promotor del concurso y las características del mismo; las bases y cuerpos de asesores y jurados, sitios y condiciones de producción, premios, testimonios, textos y teorías arquitectónicas, etc. Así, se construye una urbimbre que vincula la producción local en un contexto de apertura internacional.

En este sentido, una nueva generación de arquitectos incentivaba a reorientar la reflexión de la disciplina sobre sus tramas, traducciones y fuentes de inspiración y planteaba, en sus principios, un conjunto de propuestas en la inacabada cultura arquitectónica. Afirma Maestrepieri, que, producto de esta prolífica situación "[...] es posible percibir prefiguraciones de ciertos problemas y singularidades -rupturas y tragedias-, de los procesos politicos y sociales que atravesaron a la Argentina entre los años sesenta y setenta [...]" (MAESTREPIERI, 2004, p.26). Trazar un mapa geográfico-cultural (proceso), donde el mecanismo de concurso, como legitimador disciplinar se tensa entre las variables de la disciplina (objeto) y los modos de producción del lugar (contexto), nos posibilitando construir una micro-historia ${ }^{1}$, desde la cual se puede vislumbrar las vinculaciones entre los programas, políticas públicas e instituciones relacionadas, equipos de proyecto y lugar.

En este marco, proponemos como hipótesis de lectura que en el período que comprende los años 1959-1968², bajo el marco socio-económico del desarrollismo y por intermedio del instrumento del concurso abierto, se produjo una renovación de la arquitectura en Argentina. Para mostrar esto, examinaremos algunos concursos y proyectos puntuales. De este modo, el artículo se estructura en (I) una primera parte analítica-reflexiva, donde se presenta el ideario desarrollista como aquel que ha transformado de manera sustantiva las distintas esferas que han incidido en la práctica de la cultura general y, en particular, en los modos de hacer arquitectura. A continuación, (II) se realiza un análisis de tres (3) casos de concursos de arquitectura argentina, caracterizándolos e interpretándolos, y se intenta poner en evidencia ciertas hipótesis y lógicas de partida desde las cuales se generaron: el Instituto de Previsión Social de Misiones (Posadas, 1959), el Cementerio Parque Ciudad de Mar del Plata (Mar del Plata, 1962) y el Edificio Sede de la Unión Industrial Argentina (Capital Federal, 1968). (III) En la tercera parte, se exponen las conclusiones acerca de la implementación de los casos presentados, lo que nos posibilita elaborar un marco teórico que comprende y articula diversas variables desde las cuales se construye este significativo campo de producción de la arquitectura. Aquí, se destaca como elemento común o regla la innovación y, donde la originalidad en los resultados proyectuales es una referencia significativa en la transformación de la arquitectura argentina.

\footnotetext{
${ }^{1}$ La "micro-historia", "micro-análisis" o "micro-fenómeno", es una posición metodológico-historiográfica que se vincula con las producciones de historiadores como Carlo Ginzburg, Giovanne Levi, Edoardo Grendi o Jacques Revel. En este artículo no haremos un desarrollo explícito de dicha categoría.

${ }^{2}$ Si bien la propagación del sistema concursal se extiende hasta principios de la década del 70 (1972), se aclara que el recorte cronológico se toma en función de la casuística escogida para el desarrollo del artículo.
} 


\section{I.- DESARROLLISMO: ARTE Y ARQUITECTURA}

Se le atribuye una constante económica de relativa estabilidad a las décadas de 1960 y 1970, a partir de algunas premisas: (1) el cierre del período de sustitución fácil de importaciones; (2) crecimiento industrial orientado a benes de consumo no durables y en menor término, a los bienes durables, intermedios y de capital. Así, se inicia una etapa de desarrollo económico más sostenido y diversificado, de cambios en el uso económico de la producción industrial, crecimiento del mercado interno y de las llamadas marcas nacionales, aumento de las importaciones industriales y mayor capacidad para generar empleo de asalariados, generando así una dinamización y enriquecimiento de la economía. De este modo, se presentaba como un ideario de crecimiento interno, sostenido por los sectores sociales comprendidos en ciertas burguesías que pretendían crecer económicamente mediante un desarrollo relativamente autónomo, a partir de la nueva integración de los capitales nacionales en la etapa monopólica del intercambio capitalista, sostenido por estudios de la $\mathrm{CEPAL}^{3}$ y otros organismos técnicos del continente.

En parte, este desarrollo y el cambio en la estructura de producción fueron posibles gracias al estímulo de la Ley de Inversiones Extranjeras ${ }^{4}$ (1958) durante la presidencia de Arturo Frondizi, su implementación activó fundamentalmente la relación con los Estados Unidos. Esta posición es confirmada por la declaración de Juan Carlos Portantiero en 1977, quien sostiene que "[...] solo las inversiones de Estados Unidos ascendieron entre 1960 y 1968 de 472 millones a 1.148 millones de dólares, o sea un incremento del $243 \%$, mientras en América Latina fue del
32 \% [...]” (PORTANTIERO, 1977, apud GARCÍA CANCLINI, 1988, p.103).

En el campo cultural, perdieron poder y fueron desplazadas del centro de la escena aquellas instituciones arcaicas, representativas de la burguesía agroexportadora (Academia de Bellas Artes, la Revista Sur, el diario La Nación) y su lugar fue cubierto por el Instituto Di Tella ${ }^{5}$ y otros centros de experimentación ligados a empresas industriales. Este nuevo clima estético resultó, entre otros, en la aparición de las revistas Primera Plana, Confirmado, Los Libros, agencias de publicidad y centros de artes (Centro de Artes Visuale ${ }^{6}$, liderado por Jorge Romero Brest) que operaban como verdaderos órganos de difusión y radiación de este ideario de esperanza de modernizar el país. En este punto, nos interesa remarcar el giro cultural hacia Norteamérica por parte de Argentina y América Latina producto de diversas acciones. Por un lado, la penetración masiva de capitales industriales era acompañada en el campo cultural con subsidios de fundaciones (Ford y Rockefeller); la creación de un "[...] Consejo Internacional por parte del Museo de Arte Moderno de Nueva York en 1952 [...]” (GARCÍA CANCLINI, 1988, p.106) exportando muestras de pintura de vanguardia; también el sostén económico a museos, revistas y críticos por parte de empresas transnacionales (Esso, Standard Oil, Shell, General Motors, General Electric, etc), la Unión Panamericana, la CIA, prefiguraron una potente campaña que, por diversas vías, a veces ocultas, "[...]impulsaba un mismo proyecto: difundir una experimentación formal aparentemente despolitizada, sobre todo el expresionismo abstracto, como alternativa al realismo social, el muralismo y toda corriente preocupada por la identidad nacional de nuestros paises [...]" (GARCÍA CANCLINI, 1988, p.106).

\footnotetext{
${ }^{3}$ Con relación a la corriente desarrollista y la Comisión Económica para América Latina y el Caribe, Aníbal Jáuregui plantea que ésta se presentaba como su think tank; así como el desarrollismo fue "el modo de denominar al consenso reformista del capitalismo como respuesta a los déficit que presentaba el crecimiento económico regional" (JÁUREGUI, 2016, p.127).

${ }^{4}$ Ley N ${ }^{\circ} 14.780$ de Inversiones Extranjeras, sancionada por el Honorable Congreso de la Nación Argentina el 4 de diciembre de 1958, vinculada a la promoción industrial

${ }^{5}$ Fundado en 1960, se propone crear un equipo intelectual - teórico y cultural - capaz de dar una orientación ideológica y de ser la expresión cultural del desarrollo. (LONGONI, 2011, p.139).

${ }^{6}$ Su misión es clave: "lograr que los artistas produzcan, teniendo en la cabeza la información de los grandes centros metropolitanos para poder incluir luego a la Argentina en eje Nueva York-Paris-Tokio, centrales mundiales de la plástica de vanguardia”. (LONGONI, 2011, p.133).
} 
Completa el panorama las transformaciones sustantivas en las ciencias sociales y en las ciencias humanas como la Antropología, así como en otras que estaban en auge como la Semiología y la Teoría de la Comunicación y que tuvieron una particular incidencia en el desarrollo de la cultura y "[...] habrian de reflejarse en las ideologias arquitectónicas en la década sucesiva [...]” (WAISMAN, 1984, p.62). Destaca Waisman, la apreciable relación que se establece entre tecnología y ciencia en la que sobresale la creación de la cibernética y su teoría básica, la teoría de la información. La idea de eficacia y implementación a través de modelos serán aspectos centrales de esta metodología, que "[...] trascenderá a otros campos de estudio, entre ellos la arquitectura y el urbanismo [...]” (WAISMAN, 1984, p.61-62).

En el campo del diseño y más específicamente de la arquitectura que, al margen del cambio de escala en la producción, los proyectos se desarrollaban a partir de los grandes form - givers o concept-givers, creadores de ideas arquitectónicas. Este proceso tuvo un impacto en la renovación del lenguaje, a partir del uso de nuevos materiales (acrílico, plástico, poliéster, aluminio, aglomerados y maderas laminadas, vidrios templados, fibras de vidrio, etc), así como la profundización de la ingeniería estructural (acero y hormigón) y los nuevos procedimientos tecnológicos. Con relación al prolífico e intenso intercambio entre el inédito tipo de creatividad espacial-arquitectónica y los recursos tecnológicos disponibles, Marina Waisman describe la década del sesenta como la verdadera década vanguardista del siglo. Entendiendo el fenómeno a partir de la ruptura total con la tradición, la cultura arquitectónica se configura a partir de un panorama de extrema complejidad, donde el cambio, la innovación, la disrupción y la actividad creativa son piezas indispensables para empezar a comprender ciertos procesos de transformación la disciplina.

\section{II.1- INSTITUTO DE PREVISIÓN SOCIAL MISIONES - POSADAS (1959 - ARQS. MARIO SOTO - RAÚL RIVAROLA)}

Este concurso regional de anteproyectos y su posterior obra parten de una premisa central: el gobierno de la provincia de Misiones, en su nueva condición de estado provincial, se emparentaba con la identidad política y económica desarrollista del gobierno nacional de Arturo Frondizi, iniciado en 1958. En esta coyuntura, se habían generado algunas acciones de modernización ${ }^{7}$, dentro de un amplio conjunto de prácticas a escala territorial (Plan Regulador Provincial, obras de infraestructuras energéticas, hospitalarias, etc.) y donde se encuadra dicho concurso.

Las bases del concurso proponían un programa de necesidades y usos que, producto de lo exiguo del lote, implicaba una mayor densidad constructiva en superficie, lo que generaba un desafío proyectual en la resolución de los espacios intermedios y en la generación de formas abiertas que minimicen la rigurosidad climática. El proyecto era un complejo edificio en altura que incluía oficinas públicas del Instituto de Previsión Social de Misiones (en adelante IPSM), galerías comerciales, hotel, restaurant y confitería. Esta complejidad programática y funcional se refiere, según Roberto Fernández, a la "[...] intención de pensar una arquitectura contributiva a la urbanidad, una arquitectura-ciudad como la que por entonces teorizaba en sus clases Winograd o asimismo investigaban otros proyectistas sudamericanos contemporáneos ${ }^{8}[\ldots] ”$ (FERNÁNDEZ, 2004, p.16).

Luego del análisis de determinadas condicionantes: (1) geográfico-ambientales (clima, latitud, orientación,

${ }^{7}$ Vinculadas a otros concursos que se sucedieron entre los años 1956/57 en Misiones, donde los arquitectos Rivarola y Soto fueron los ganadores, como ejemplo la Escuela Normal $\mathrm{N}^{\circ} 1$ de Alem y las Hosterías de El Dorado, Apóstoles y San Javier.

8 “Arquitectos como Levi, Vilanova Artigas, Reidy o Moreira Salles, que en una clave híbrida de racionalismo y neoexpresionismo hormigonero no sólo reelaboraban el legado tardomoderno corbusierano sino que se abocaban a la construcción del equipamiento social, en una escala, magnitud y calidad por entonces desconocida en Europa o Estados Unidos" (FERNÁNDEZ, 2004, p.14-15). 


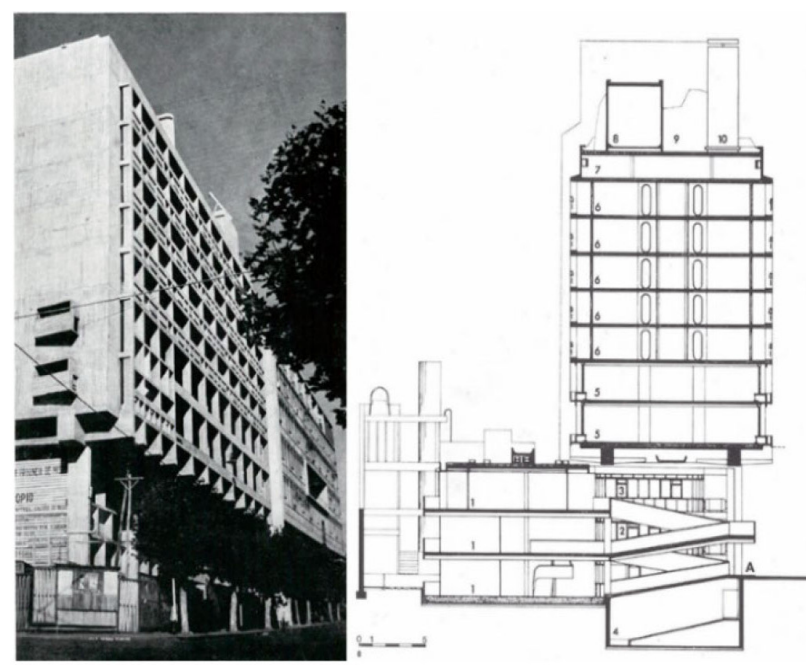

Imagen 1. Perspectiva urbana. Vista desde la esquina de Junin y Bolivar, continuidad y conexión formal con el edificio del Correo. Corte Transversal que define los accesos y la articulación volumétrica con el espacio público.

Fuente: Revista Summa No 8. Año: 1967

regímenes de lluvia, etc.); (2) antropológico-culturales (conductas humanas), (3) características urbanoarquitectónicas presentes en la ciudad: construcciones bajas en un solo nivel, con un profundo paisaje verde frente al río Paraná, sin la tradición de la arquitectura colonial característica de otras ciudades del noreste argentino. En respuesta a esto, los autores proponen una serie de estrategias proyectuales que se valen del reconocimiento del manejo del sitio: (1) la reconstrucción de la calle, (2) la porosidad de la manzana vista a través de los accesos y el patio urbano. El resultado es simple y "[...] puede ser leída en clave de basamento y bloque, donde la "plataforma" de entendimiento y diálogo urbano, el lugar del encuentro social, es el zócalo "denso" de la ciudad, la calle definida desde el espacio público que conformaban las hasta entonces menospreciadas fachadas de la ciudad real [...]” (MAESTRIPIERI, 2004, p.53).

Alvaro Arrese valora el grado de acierto en la ubicación del núcleo vertical, al trazado como un fuelle acústico entre las habitaciones del hotel y la articulación con el edificio contiguo del Correo?. Asimismo, manifiesta la

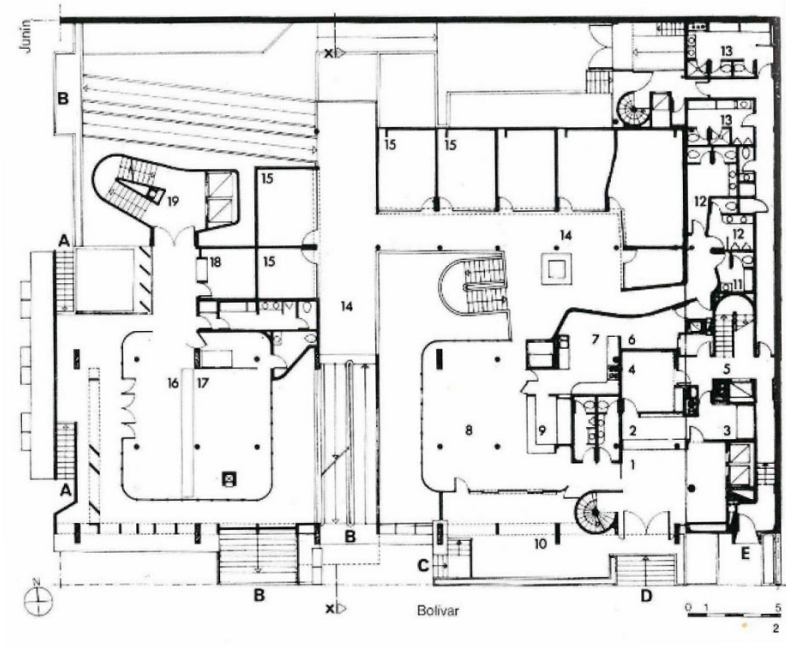

Imagen 2. Planta Baja (nivel + 1.40 mts.) Edificio IPSM. Se destaca el manejo de las proporciones, la escala humana, el espacio dentro de otro espacio, a través del diseño de elementos fijos de equipamientos, la fuerza en relación a la comprensión del sujeto como un ser existencial. Fuente: Revista Summa $N^{\circ}$ 8. Año: 1967

clara diferenciación en la comprensión y valoración de los aspectos urbanos-ambientales entre ambos equipos de proyecto, ya que mientras el edificio del Correo "[...] opone al sol misionero livianas fachadas vidriadas y cortinas venecianas interiores para regular su paso, el hotel antepone a la vidriera robustos balcones y parasoles de hormigón para proteger sus vidrios [...]" (ARRESE, apud MAESTRIPIERI, 2004, p.52).

Otra estrategia significativa de interacción con el entorno ha sido la múltiple y correcta articulación volumétrica entre el cuerpo principal (bloque alto) y los cuerpos menores, prefigurando un patio abierto a la calle lateral, relacionando este conjunto con el tejido posterior de menor altura. La variedad espacial, comenta la Arq. Myriam Goluboff es vista "[...] a través de elementos tan puramente arquitectónicos como el manejo de los volúmenes, las proporciones y la luz, con el apoyo de la geometría, de la proporción raíz de dos y de una fruición por los trazados de la que nos queda testimonio, entre otros, en el diseño de la torre de circulaciones del IPSM [...]" (GOLUBOFF, 2004, p.20). 
Imagen 3. Vista de la fachada desde la calle Junin hacia el patio interior. El juego de gárgolas, balcones, rampas y terrazas se une a las palmeras para conferirle al edificio su carácter netamente tropical. La torre de circulaciones del hotel actúa como elemento de transición entre el volumen del IPSM y el del edificio del Correo.

Fuente: Revista Summa No 8. Año: 1967

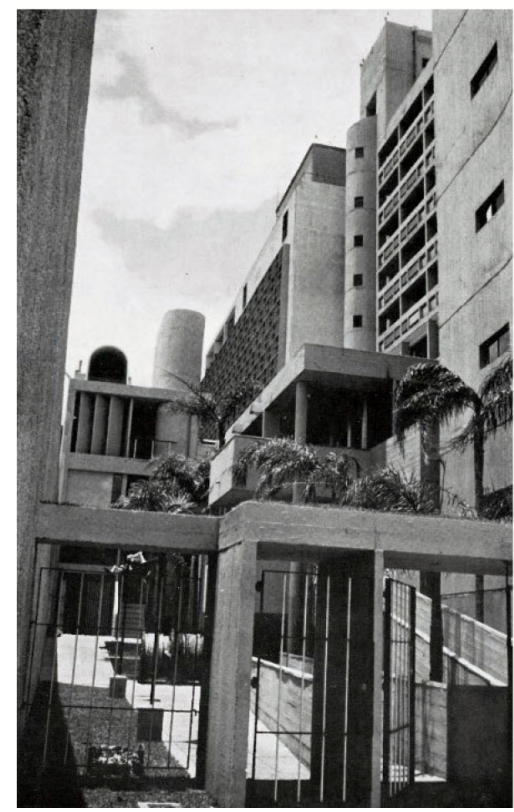

Imagen 4. El uso intensivo del hormigón y la ductilidad del material, creando espacios intermedios, en este caso encerrando el hall de entradas. Arrese señala que más allá de la influencia de Le Corbusier, bay una notable contemporaneidad con otros arquitectos, como Kenzo Tange o Josep

Luis Sert.

Fuente: Revista Summa No 8. Año: 1967

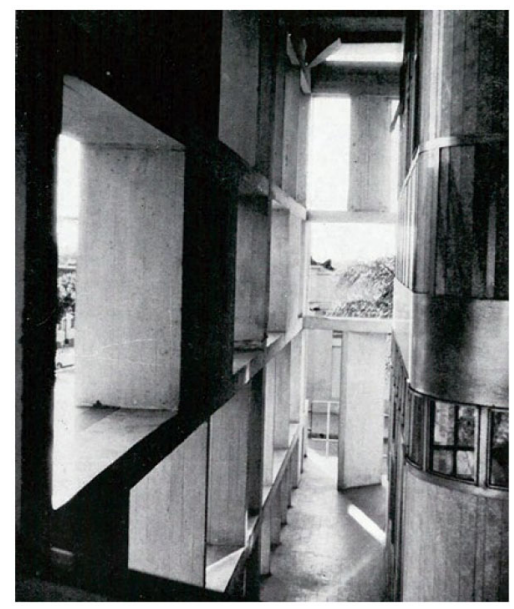

Uno de los objetivos del proyecto fue remarcar la identidad nacional en los concursos. En este sentido, es relevante el modo en que se presenta en la Revista Summa $\mathrm{N}^{\circ} 2^{10}$ : allí se pone énfasis en la significatividad de esta obra en tres aspectos: (1) el tratamiento fluido del espacio, (2) el manejo de la iluminación y (3) la valorización de la naturaleza de los materiales (Imagen 4), reafirmando la atención a las necesidades de las comunidades del interior del país, confirmando su aporte creativo, generacional, que se traduce en algo argentino y, quizás también latinoamericano.

Como es conocido, a fines de los años 50, Misiones se conformaba por una sociedad nueva, plural, integrada por inmigrantes de muy diversos países europeos. Por ello, la construcción de una nueva tradición para la nueva sociedad, conforma el marco en el que debe interpretarse la exploración de la forma arquitectónica en la temprana obra de Rivarola y Soto. Estas imágenes, donde la construcción y búsqueda de una identidad cultural reconocen "[...] desde una invención de arquitectura contemporánea y sincretista hasta la ficción de una invasión extraterrestre ${ }^{11}$ " (MAESTRIPIERI, 2004, p.30), la importancia y la incidencia del contexto del paisaje natural o cultural como "[...] determinante de la implantación y como modulador y mediador de la escala del edificio [...]” (MAESTRIPIERI, 2004, p.32). También, el abandono de las metáforas mecánicas, acercando la propuesta a las producciones más artesanales del período. En este sentido, Maestripieri reconoce en Mario Soto como en Le Corbusier que:

Uno de los rasgos importantes del siglo $\mathrm{XX}$ es haber puesto a la técnica al servicio de la arquitectura con una libertad nunca antes alcanzada. Esta libre disponibilidad significaba adaptarse a las condiciones locales de producción [...] se vale tanto de la alta tecnología como de técnicas artesanales y como su maestro Traine, recurre y adapta técnicas utilizadas por otras disciplinas y evita caer en la pura estética o expresionismo de la técnica (MAESTRIPIERI, 2004, p.32).

\footnotetext{
${ }^{10}$ Dirigida por Carlos Méndez Mosquera, editó su primer número en abril de 1963 y "buscaba posicionarse como un medio de comunicación innovador y propositivo" (MAESTRIPIERI, 2004, p.42)

${ }^{11}$ Se refiere a la historieta El Eternauta, en donde Buenos Aires había "encontrado un nuevo espacio visual, una identidad cultural que no era pictórica, ni literaria. (MAESTRIPIERI, 2004, p.30).
} 


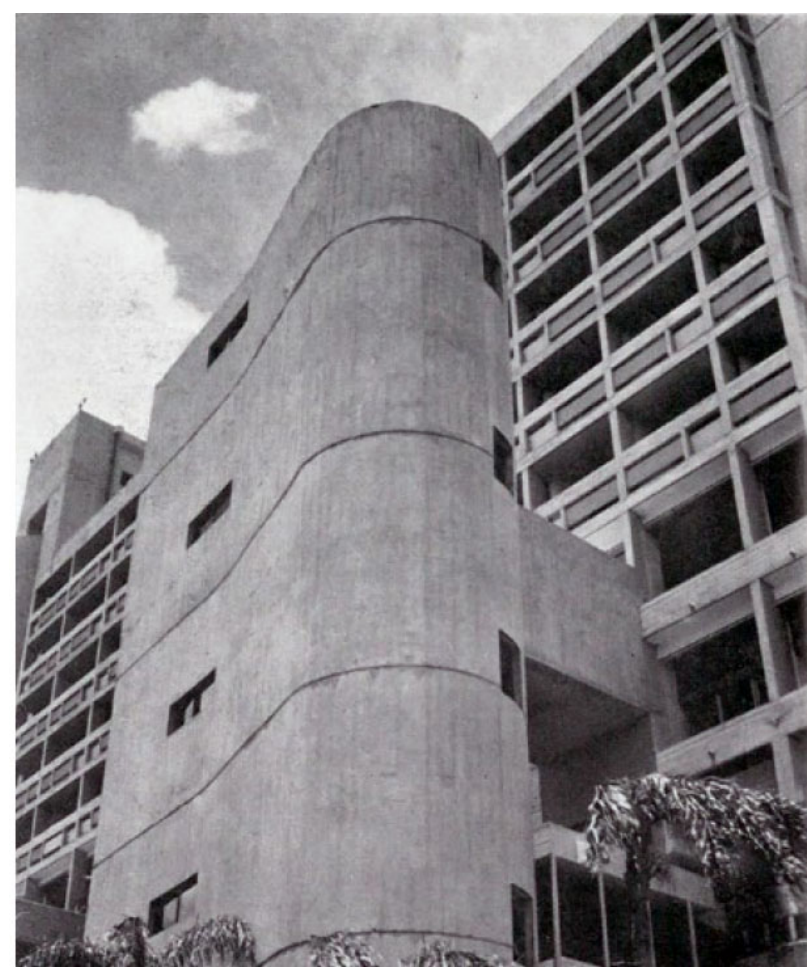

Imagen 5. Torre de circulación edificio IPSM.

Fuente: Revista Summa No 8. Año: 1967

Para finalizar: si, como plantea Roberto Fernández, la tecnología es un soporte de identidad regionalista que construye una "[...] geoculturalidad especifica [...]" (FERNÁNDEZ, 1998, p.232), las condiciones de producción y las tecnologías posibles definen, dentro de un amplio espectro de variables, la producción arquitectónica y urbana. Entendemos que los autores proponen, precisamente, esta "geoculturalidad" en este dispositivo proyectual, dado que, en particular, estimula visiones renovadas en la forma de pensar y construir estos condensadores sociales. Esto se ve reforzado a partir del contrapunto producido por la síntesis que se genera entre la heterogeneidad étnica misionera, la naturaleza, cruzada por la geografía y la historia y los sueños utópicos de una modernidad inacabada, en búsqueda de la esperanza de alumbrar lo nuevo, la tradición moderna.

\section{II.2- CEMENTERIO PARQUE CIUDAD DE MAR DEL PLATA (1962 - ARQS. HORACIO BALIERO - CARMEN CÓRDOVA DE BALIERO)}

Concurso de anteproyectos de carácter nacional para el Cementerio Parque de la Ciudad de Mar del Plata (CPMDP), se enmarca dentro de un proyecto integral de carácter paisajístico. Los autores planteaban que el mismo estuvo "[...] muy inspirado en arrozales[...]" (SCHERE, 2008, p.287), al estar proyectado e integrado a las curvas de nivel que presentaba la topografía del lugar.

En la formación de H. Baliero, el entendimiento de las ciencias básicas parece ser interesante para comprender el desarrollo de su obra. La claridad de conceptos lo lleva a tener una gran capacidad de análisis y a organizar sus pensamientos, utilizando como herramientas primarias a la topología, la geometría y la matemática, para proyectar, trabajar en obra, en la corrección de concursos o en la actividad docente. Además, resulta fundamental la idea y conformación del grupo (la prédica de Walter Gropius a favor del trabajo en equipo) Organización de Arquitectura Moderna (OAM) ${ }^{12}$, formado por gente y amigos con habilidades complementarias. Sobre los jóvenes de la OAM, plantea Liernur:

Formaban parte de una vasta corriente que agitaba a un sector de los intelectuales argentinos, disconformes con los rumbos adoptados en el país, críticos del pasado conservador, partidarios de políticas de modernización estructural y cultural, partícipes de la pérdida de confianza en el progresismo modernista - industrial y estético - provocada por la Segunda Guerra Mundial (LIERNUR, 2004, p.115).

\footnotetext{
${ }^{12}$ El Grupo OAM (Organización de Arquitectura Moderna), relacionados a la corriente filosófica Existencialista, era un espacio de intercambio y movilidad cultural donde se mantenían intereses comunes por la arquitectura y la permanente lucha contra la enseñanza del academicismo en la facultad.
} 
Imagen 6. Planta general del CPMDP donde en el centro se articulan un grupo de construcciones simples alrededor de la playa con la explanada y el techo para homenajes. Completan los elementos construidos el crematorio y el Panteón Municipal, fundidos al entorno natural por medio de taludes de césped o plantas.

Fuente: Baliero. Año: 2006

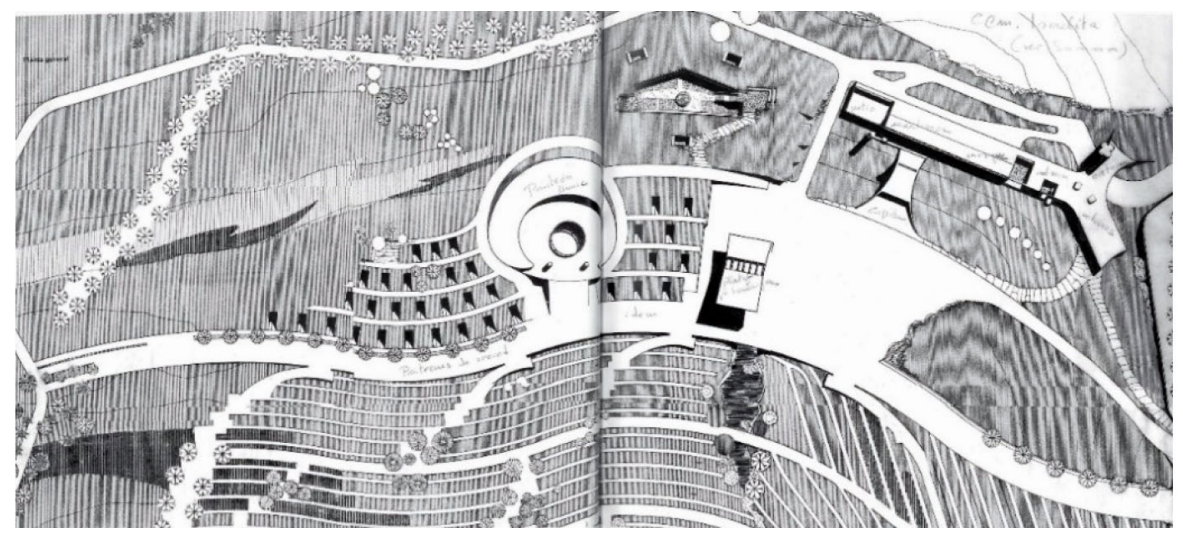

Las convicciones e ideales que particularmente nutrieron el universo de Baliero tuvieron su momento de mayor intensidad en los concursos y obras del CPMDP, en el Cementerio Israelita de Mar del Plata y en el Colegio Mayor Argentino de Madrid ${ }^{13}$ (1964). Se puede afirmar, según Liernur, que fue cautivado por tres modelos referentes: (1) la obra del Grupo Austral y en particular Antonio Bonet, (2) las referencias a Richard Neutra y (3) la arquitectura brasileña ${ }^{14}$; a lo que se puede agregar los propios comentarios del autor con relación al nivel de información con que se contaba sobre publicaciones y revistas nacionales ${ }^{15} \mathrm{e}$ internacionales que marcaban y entusiasmaban ciertos rumbos. A partir de estas traducciones conceptuales y sumado la producción material en "[...] particular la arquitectura holandesa, el edificio de la Baubaus en voladizo sobre su base negra, y un edificio de Hans Wittwer, que pocos conocen: el pequeño aeródromo de Schkeudits. [...]” (DAITCH, 2006, p.18), se puede construir un cuerpo de pensamiento en el que las producciones humanas, el arte y la técnica tienen una articulación coherente dando respuestas a las condiciones de "[...] reproductibilidad y al compromiso social [...]" (LIERNUR, 2004, p.116) constituyéndose en pilares desde donde Baliero sostenía su arquitectura.

El planteamiento proyectual para el CPMDP se fusiona con el paisaje, con el lugar. Su acceso principal se ubica en una altura media con respecto de las alturas totales del sitio, dando como resultado que la mitad del terreno esté cuesta abajo y la otra mitad en pendiente hacia arriba (Imagen 6). La composición general de las distintas construcciones que la integran, genera un contraste producto de su materialidad, siendo estos "[...] fuertes elementos plásticos de color blanco [...]" (BALIERO/CÓRDOVA, 1968, p.41).

$\mathrm{Al}$ respecto dice Baliero que "[...] las grandes formas blancas y curvas de los distintos edificios no buscan más que reforzar y poner en valor los datos ya implícitos en el paisaje natural [...]" (DAITCH, 2006, p.136). Estas emergen naturalmente, o por generación puramente plástica, pero con un sentido estricto en el mecanismo funcional y constructivo. Expresa Baliero, sosteniendo un profundo pragmatismo: "[...] en definitiva todo lo que hace de la obra una totalidad orgánica es función [...]” (BALIERO, opud DAITCH, 2006, p.141). La arquitectura del conjunto radica en la síntesis y potencia que cobran unos pocos elementos puestos y desde el propio sitio; por un lado, el pórtico de hormigón de entrada con una cubierta de cuatro patas por donde cae el agua de lluvia, por otro, se encuentra el Cementerio Israelita (Imagen 7), una pequeña obra doméstica, "[...7 consistente, en lo esencial, en dos paredes curvas que no se tocan, y cuyos desencuentros producen el momento de entrada

\footnotetext{
${ }^{13}$ Las estrategias de proyecto del concurso en Madrid y en concordancia con el CPMDP, Baliero recuerda que en cuanto estudió las bases del programa, observó la planimetría del terreno y las fotos del mismo, vislumbró la idea del proyecto: “esto es una hoya igual que Mar del Plata” (DAITCH, 2006, p.111).

14 “Con la arquitectura del Brasil, la curva se incorporó al repertorio de Baliero como rasgo definitorio, aunque no obsesivo, de su lenguaje” (LIERNUR, 2004, p.116).

15 "Baliero fue miembro fundador y del comité de redacción de la Editorial Nueva Visión (1950)" (LIERNUR; 2004, p.115).
} 


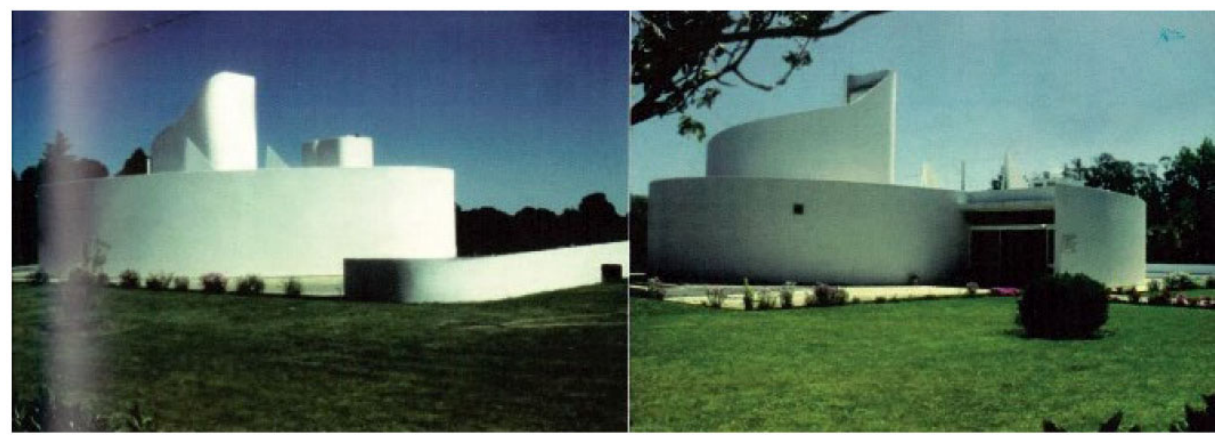

Imagen 7. La forma del Cementerio Israelita surge contenida por muros de igual altura, referida y determinada por su basamento. Un techo plano, que se va elevando, se asienta sobre los muros. Los autores trataban de crear un espacio apto para realizar un recorrido con pausas, donde las plegarias y los cánticos confluyan en interiores caracterizados por una unidad plástica, el revoque salpicado blanco.

Fuente: Baliero. Año: 2006

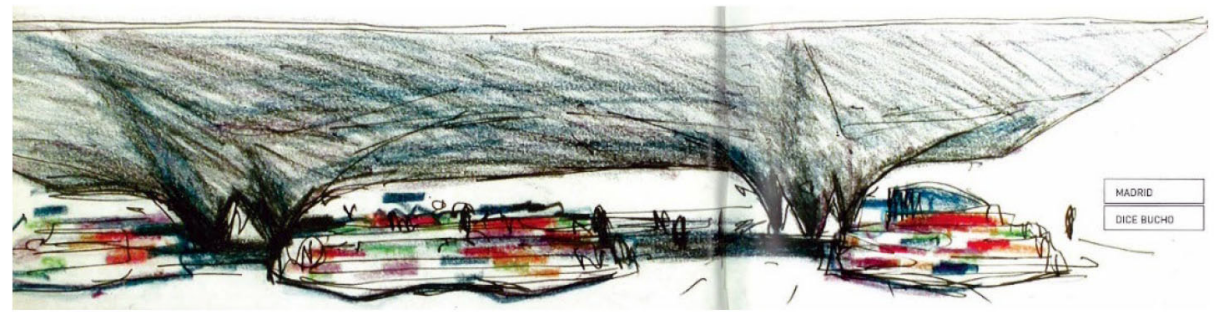

Imagen 8. Dibujo de la estructura de bormigón que alberga los puestos de flores realizado por Baliero.

Fuente: Baliero. Año: 2006

y el momento de salida [...] inmersa en ese espacio descomunal [...]” (DAITCH, 2006, p.137). Aquí, se destaca el sentido y correcto manejo de la escala en varios órdenes, procurando que la buena arquitectura pueda vivenciarse desde las visiones próximas y lejanas.

Completan la propuesta y la experiencia, la gran explanada para el público, los quioscos de flores, la playa de cortejos, el crematorio, el Panteón Municipal, la Explanada de Homenajes, las bóvedas y enterratorios enlazados al paisaje a través de ejes de composición en donde los recorridos y senderos son sugerentemente acompañados por una esquemática estructuración de líneas de árboles y plantas (araucarias, jacarandás, sauces, cañas, abedul, liquidámbar, entre otras) generando diversas texturas, colores y olores.

Entendiendo a la arquitectura como una experiencia, Martin Heidegger señala que la existencia del ser se realiza en el lugar arquitectónico que habita, "[...] ya que habitar es la manera en que los mortales son en la tierra, es la manera de ser del hombre [...] construir, habitar y pensar forman parte de los mismos hechos definitorios de la existencia del hombre" (HEIDEGGER, opud MONTANER,
1993, p.65). También Louis Kahn sintonizaba con estas ideas, comenta Montaner, podríamos pensar que Baliero y Córdova se anticipaban con sus ideas y proyectos y parafraseando a Montaner, la arquitectura del CPMDP sería:

Una serie de formas geométricas ordenadas creadas artificialmente por el hombre definiendo entre ellas - la forma - el suelo - la tierra, un intersticio, un minúsculo espacio existencial, una burbuja confortable - la arquitectura -, donde habita el hombre [...] con ello se realiza la ambición de Kahn de crear con la arquitectura un mundo dentro del mundo (MONTANER, 1993, p.65).

Baliero y Córdova con inteligencia y creatividad, crearon un mundo para la trascendencia para aquellos que no habitan más el mundo de los mortales y su enseñanza reside "[...] en las cosas sencillas, precisamente en la secreta y modesta complejidad [...]" donde "[...] el bosque era una cultura, otra [...]" (CHERNY, 2004, p.111), donde la arquitectura como construcción cultural de espacios habitables se hará con una estética de lo necesario. 


\section{II.3- EDIFICIO SEDE UNIÓN INDUSTRIAL ARGENTINA (UIA) - CAPITAL FEDERAL (1968 - ARQS. FLORA MANTEOLA, JOSEFA SANTOS, IGNACIO PETCHERSKY, JAVIER SANCHEZ GÓMEZ, JUSTO SOLSONA Y RAFAEL VIÑOLY)}

El concurso de carácter nacional para la sede de la UIA, que se ubica en los terrenos de Catalinas Norte, área que tiene cierta especificidad e historia dentro de la ciudad de Buenos Aires. Allí el código de edificación contenía una reglamentación especial que, estipulaba limitaciones conrespecto a la ubicación, dimensiones en los lotes, entre otros.

Este proyecto se enmarca dentro del proceso de concentración de los roles económicos auspiciado e iniciado por el desarrollismo. Considerando esta lógica, Adrián Gorelik comenta:

Los años sesenta, el centro estaba afianzando su carácter terciario, con una verticalización que comenzaba a justificar en el plano edilicio la jerarquía que Buenos Aires ya tenía en el poblacional (en 1967, con un área metropolitana de más de 8 millones de habitantes, compartía con Paris el tercer lugar entre las metrópolis mundiales, detrás de Nueva York y Tokio y delante de Londres) (GORELIK, 2016, p.329).

Esta concepción de la planificación desarrollista de densificar y volver al centro de la ciudad capital, es estimulada e impulsada por los capitales privados y acompañada por las políticas públicas del estado, reafirmando su carácter multifuncional, favoreciendo su crecimiento en altura, alentando los rascacielos para oficinas. De este modo, ajustándose a las premisas reglamentarias del código de edificación, se proponía un edificio donde el tema arquitectónico era la tipología en torre.
La propuesta ganadora evidencia un particular manejo en este tipo de proyectos, ya que el equipo había realizado una permanente experimentación formalespacial; comenzando desde el diseño de la planta, la piel envolvente o la estructura, mostrando una gran aptitud en la generación de formas arquitectónicas. Fernando Aliata comenta, sobre los modos en la construcción de estrategias proyectuales por parte del estudio, donde interactúan nuevas y antiguas teorías y que tiene un denominador común en sus esquemas o metodología proyectual que es "[...] caracterizada por el uso de la idea de partido como modo de generar una organización clara del programa funcional [...]”" (ALIATA, 2007, p.11). El partido, como lógica organizacional, es entendido por estos jóvenes arquitectos emergentes de la universidad luego de $1955^{16}$, sumidos en el inconformismo y rechazo a la modernidad ortodoxa, como una "[...] herramienta progresiva de transgresión en la cual el uso de la analogía y la acción gestual, propias de las vanguardias pictóricas, se convierten en instrumentos auxiliares fundamentales [...]" "[...] el desafío es la inversión de significados [...]" (ALIATA, 2007, p.12). En este caso, esta estrategia puede vislumbrarse en "[...] la transparencia del curtain wall, que pone en cuestión la división tripartita de la tipología del rascacielos en la sede de la UIA [...]" (ALIATA, 2007, p.13)

La estrategia de partido se sintetiza en tres elementos (Imagen 9): “[...] un envase de cristal que envuelve las oficinas, un prisma de hormigón que contiene los núcleos verticales y un sistema de cajas metálicas que unifica los servicios, ya sea los ubicados en el basamento o los de la misma torre [...]” (AAVV, 2007, p.95). La preocupación y el interés en estos temas proyectuales se centran para Solsona a partir de la "[...] libertad formal que permite como por lo que impone, tanto las viviendas como las oficinas en altura constituyen en sí mismas, y de muy diversos modos, piezas urbanas capaces de adquirir autonomía formal [...]" (SOLSONA, 1997, p.123). El jurado del concurso valora en su dictamen las virtudes del proyecto ganador. En efecto, destaca el aporte que realiza al nuevo panorama arquitectónico a través de la

16 “Punto de inflexión en la enseñanza e implementación de los programas y planes de estudio de la Facultad de Arquitectura de Buenos Aires" (BERTOLI, 2003, p.17). 


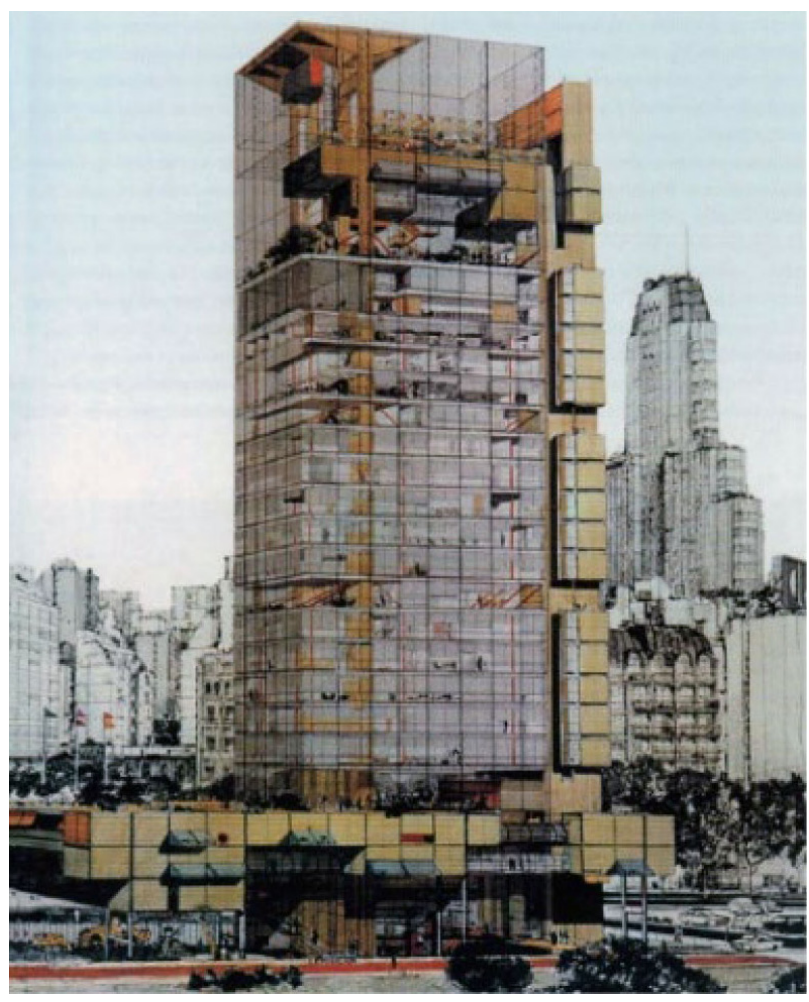

Imagen 9. Perspectiva urbana de concurso.

Fuente:wnw.academia.edu/7968986/Concurso_de_la_UNION_INDUSTRIAL_ARGENTINA. "El cuerpo de la torre se desarrolla de acuerdo con la solución tradicional de losas superpuestas que conforman espacios estratificados hasta el último nivel de oficinas, pero a partir de éste, aun cuando el envoltorio perimetral vidriado continúa sin modificarse, dentro del volumen se produce un cambio fundamental: los locales, encerrados en cajas o ubicados sobre planos que juegan libremente en su relación espacial, se intercomunican a través de tubos transparentes, rampas, escaleras, hasta darnos la sensación de que nos encontramos frente a un fascinante mecanismo de relojería encerrado en un fanal" (AAVV, 1969, p.80).

imaginativa relación de la disposición de la torre y el basamento:

Organizaciones espaciales más libres, estructuración de los volúmenes en diversos planos [...] dinamismo...mediante la ubicación de la batería de ascensores sobre una de las paredes exteriores...transparencia de la caja [...] la torre llega con independencia y clara definición hasta el nivel de tierra, rodeada por el basamento que se estructura como aro independiente y da amplia interpenetración interior - exterior. Buena zonificación del edificio, en vertical, definido tratamiento exterior y homogénea solución estructural (AAVV, 1969, p.80).

Esta estrategia se logra a partir de emerger la torre desde el nivel - 2.00 metros y se mantiene así en toda su altura, este anillo tracciona como basamento sin generar interferencias a la continuidad del volumen prismático principal de vidrio. La libertad con que se estructura la propuesta, se hace presente ya en el diseño del basamento, potenciado a partir de la complejidad que presenta el programa funcional:

Un salón de actos con capacidad para (600) seiscientas personas, un club, otros..., interjuegan y cubren en parte con sus planos de cristal y acero enlozado la gran plataforma de exhibición de productos y maquinarias ubicada a tres metros bajo el nivel del terreno, pero abierta a las visuales desde el nivel peatonal. Esta plataforma de exhibición abarca toda la superficie del terreno y se convierte, por lo tanto, en el motivo central de la planta baja definiendo claramente el carácter del edificio (AAVV, 1969, p.80).

Con relación a la planta tipo de oficinas (Imagen 10), fue proyectada para albergar $19.800 \mathrm{~m} 2$ y se estructura a partir de cuatro puntos internos que operan como una estructura de apoyo continua que, sumado a la ubicación del núcleo vertical y de servicios orientados al oeste, optimizan el rendimiento y brindan una completa flexibilidad de uso. Estructuralmente y, remarcando la gran libertad de distribución que posee la planta libre, comenta el autor, que "[...] cada cuatro pisos hay una viga de arriostramiento, que vincula los cuatro apoyos de hormigón. A su vez, de esas vigas cuelgan tres pisos, por medio de tensores, mientras otras columnas apoyan en tres niveles [...]" (SOLSONA, 1997, apud ACUÑA, 2007, p.189).

La torre como tipología urbana establece una nueva trama sobre la ciudad regular y construye un nuevo paisaje estético y social dentro y sobre la ciudad 


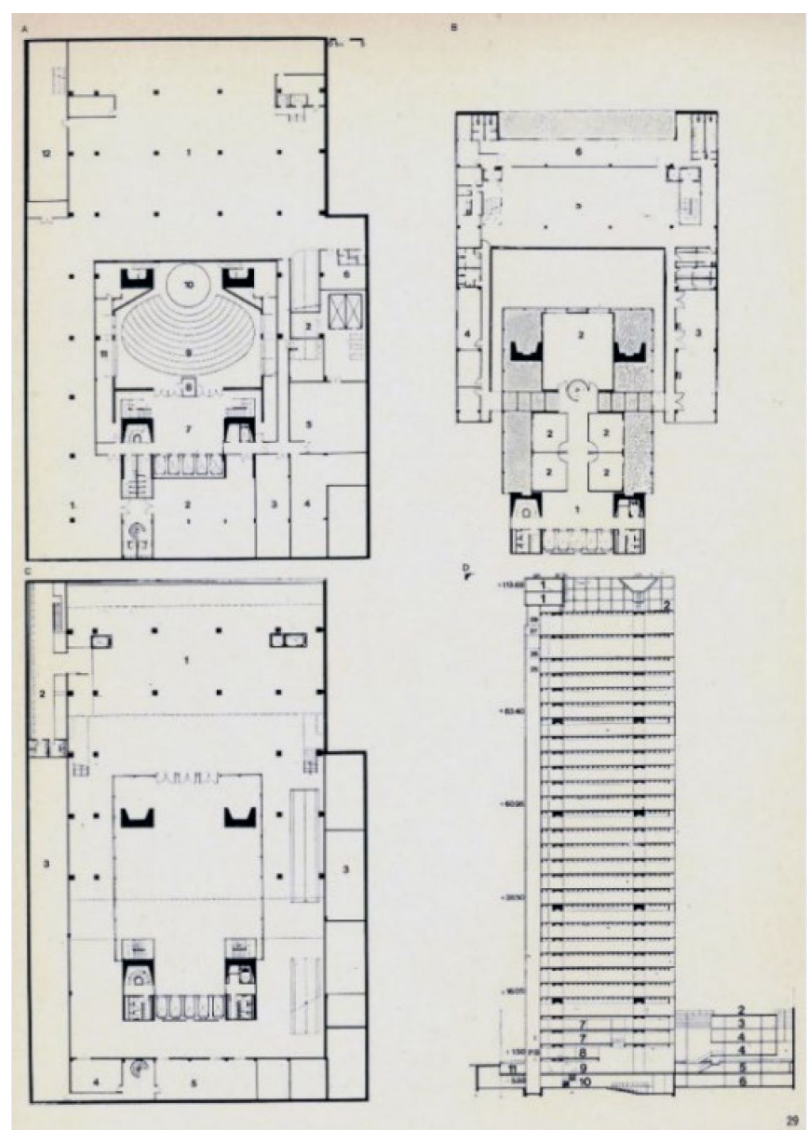

Imagen 10. Planimetría del edificio sede de la ULA. Planta del auditorio (nivel $-5.85 \mathrm{~m}$.) Planta del restaurante y de la sala de reuniones (nivel + 8.50 m.), Planta exposiciones (nivel $+11.15 \mathrm{~m}$.) y Corte.

Fuente: Revista Summa No 96. Año 1975

horizontal. Al respecto, Solsona propone el concepto de campo de intensidad, aquel que representa al fenómeno de concentración de cultura urbana, por las multiplicidades de actividades que esta genera, ya que produce un contrapunto y una alternativa real al concepto de ciudad tejido. Son premisas en las estrategias proyectuales del estudio, a partir de la conciliación de la idea de partido, verificada en: "[...] la claridad de la planta, la espacialidad difusa, la fusión con el suelo y uso de la diagonal explicita [...]”" (ALIATA, 2007, p.13), lo que confluye en un experimentalismo radical y permanente creatividad. Asimismo, podemos considerar que la trascendencia en la propuesta para la UIA está en su capacidad de diálogo con su entorno, dando respuestas en un sentido ético y estético y que parte de la "[...] reinvindicación de la arquitectura como un arte público [...]” (ALIATA, 2007, p.14).

\section{III- CONCLUSIONES FINALES}

Odilia Suárez sostiene que, para el período abordado en el presente artículo, la sinergia de los concursos " [...] se constituye en una buena dialéctica de la progresiva evolución del diseño arquitectónico en nuestro país y además colaboran, por hoy, en mantener la capacitación de nuestros profesionales cuyas energías, paradójicamente, superan las posibilidades que el país les brinda [...]” (SUÁREZ, 1972, p.96). Asimismo, durante el desarrollo del mismo hemos podido verificar la hipótesis que sirvió de guía a partir de las relaciones generadas entre las circunstancias socio-política-económica y culturales, producto de un contexto de gran complejidad y especificidad nacional e internacional (desarrollismo) y determinados proyectos que desde el propio campo disciplinar han condensado una arquitectura imaginativa y crítica.

De este modo, el estudio particularizado de cada caso, nos permite generar un corpus reflexivo acerca de la práctica de los concursos como mecanismo a partir de la obtención de los primeros premios por parte de una generación joven que desafiaba a la modernidad ortodoxa. Así, este mapeo nos posibilita construir determinados rasgos identitarios acerca de cómo esta práctica proyectual transformó el espacio arquitectónico y entonces llegar a:

a. Ser laboratorios de ideas, presentando la oportunidad y el desafío de estudiar temas o programas ciertamente interesantes y desafiantes, que bajo determinados patrones y premisas que se redactan en las bases operan como un soporte unificador e igualador de oportunidades.

b. Ser mecanismo de competición amplia y democrática, permitiendo que jóvenes arquitectos tengan la posibilidad de abordar temas y programas que no les fueron posibles mediante la contratación directa.

c. Ser un dispositivo de simultaneidad operativa, en la cual su implementación y éxito dependen de que todos los engranajes que comprenden su rueda, funcionen de manera correcta, cerrando en el óptimo resultado - concurso/obra. 
d. Ser plataforma crítica - discursiva, generando un cuerpo específico de ideas originales, donde la triada forma - función - tecnología, responde a diversos patrones en diferentes dimensiones operativas.

e. Ser mecanismo anticipatorio de proyectación, que puede operar a partir de la manifestación de nuevas resoluciones arquitectónicas sobre temas ya abordados históricamente (Ej. Tipología en torre Concurso UIA).

f. Ser un campo de experimentación proyectual, en donde se evidencian las resoluciones notables en la innovación y originalidad en sus respuestas, motorizando nuevos escenarios y desarrollos urbanos.

g. Ser soporte de oficio, entendido como parte de la formación profesional de jóvenes generaciones que comenzaron a operar en la práctica proyectual y laboral.

h. Ser un instrumento de quiebre y fragmentación de las corporaciones y relaciones de trabajo.

\section{BIBLIOGRAFIA}

AAVV. Instituto de Previsión Social, Misiones: Raúl Rivarola, Mario Soto, arquitectos. In: AAVV. Revista SUMMA $N^{\circ}$ 8. Buenos Aires: Ediciones Nueva Visión S.A.I.C., 1967. p. 39-47.

AAVV. Proyecto para la sede la Unión Industrial Argentina. Primer Premio. In: AAVV. Revista SUMMA N ${ }^{\circ} 16$. Buenos Aires: Ediciones Summa, 1969. p. 80.

AAVV. Concurso Unión Industrial Argentina. In: AAVV. Revista SUMMA N ${ }^{\circ}$ 96. Buenos Aires: Ediciones Summa. S.A, 1975.

AAVV. UIA. In: JURADO, M. (comp.). 03. Diez Estudios Argentinos. Manteola, Sanchez Gómez, Santos, Solsona, Sallaberry. Buenos Aires: AGEA. 2007. p. 94-99.

ACUÑA, V. (comp.). Solsona, Justo Jorge: Hacer y decir. 1. ed. Buenos Aires: Ediciones Infinito, 2007. 344 p.

ALIATA, F. La construcción de una estrategia proyectual. In: JURADO, M. (comp.). 03. Diez Estudios Argentinos. Manteola, Sanchez Gómez, Santos, Solsona, Sallaberry. Buenos Aires: AGEA, 2007. Prólogo, p. 10-14.
BALIERO, H; CÓRDOVA DE BALIERO, C. Cementerio Israelita en Mar del Plata. Horacio Baliero, Carmen Córdova de Baliero, arquitectos. In: AAVV. Revista SUMMA No 13. Buenos Aires: Ediciones Summa, 1968. p. 41-42.

BERTOLI, S. Década de 1950. La irrupción de la modernidad en la Facultad de Arquitectura. Buenos Aires. 2003. Disponible en: www.archivosdar.com.ar/entrevistas/ decada50.pdf. Acceso en: 28 enero 2017.

CHERNY, R. Bucho, el bosque. In: AAVV. Revista Summa + $N^{\circ}$ 66. Buenos Aires: Donn S.A, 2004. p. 111.

DAITCH, A. (coord.). Baliero. 1. ed. Buenos Aires: Facultad de Arquitectura, Diseño y Urbanismo de la Universidad Nacional de Buenos Aires, 2006. 163 p.

FERNÁNDEZ, R. El laboratorio americano. Arquitectura, Geocultura y Regionalismo. 1. ed. Madrid: Editorial Biblioteca Nueva, 1998. 316 p.

FERNÁNDEZ, R. Pasiones materiales. In: MAESTRIPIERI, E. España y la Argentina en la Arquitectura del Siglo XX: Mario Soto. Buenos Aires: Sociedad Central de Arquitectos, 2004. Obertura, p. 13-17.

GARCÍA CANCLINI, N. La producción simbólica. Teoría y método en sociología del arte. 4. ed. México DF: Siglo Veintiuno Editores, Sa de cv, 1988. 163 p.

GOLUBOFF, M. Mario Soto, la coherencia de una idea. In: MAESTRIPIERI, E. España y la Argentina en la Arquitectura del Siglo XX: Mario Soto. Buenos Aires: Sociedad Central de Arquitectos, 2004. p. 18-23.

GORELIK, A. Buenos Aires. La ciudad y la villa. Vida intelectual y representaciones urbanas en los años $1950 \mathrm{y}$ 1960. In: GORELIK, A.; AREAS PEIXOTO, F. (comp.). Ciudades sudamericanas como arenas culturales. Artes y medios, barrios de élite y villas miseria, intelectuales y urbanistas: cómo ciudad y cultura se activan mutuamente. Buenos Aires: Siglo XXI Editores, 2016. Parte IV, p. 324-345.

JÁUREGUI, A. P. Juan José Real, un comunista en la corte del Rey Arturo. Marxismo, desarrollismo y teoría del progreso en un país periférico. In: JÁUREGUI, A. P.; CERRA, A.; YAZBEK, S. Génesis y construcción del desarrollismo argentino. Buenos Aires: Biblos, 2016. p. 127-148.

LIERNUR, J. F. Baliero, Horacio. In: LIERNUR, J. F.; ALIATA, F. (comp.). Diccionario de Arquitectura en la Argentina A/B. Buenos Aires: AGEA. 2004. p. 115-118.

LONGONI, A. (ed.). El deseo nace del derrumbe. Roberto Jacoby. Acciones, conceptos, escritos. 1. ed. Barcelona: Ediciones de 
La Central en colaboración con: Madrid: Museo Nacional Centro de Arte Reina Sofía, Buenos Aires: Adriana Hidalgo editora y Red Conceptualismos del Sur, 2011. 504 p.

MAESTRIPIERI, E. España y la Argentina en la Arquitectura del Siglo XX: Mario Soto. 1. ed. Buenos Aires: Sociedad Central de Arquitectos, 2004. 208 p.

MONTANER, J. M. Después del Movimiento Moderno. 1. ed. Barcelona: Editorial GG, 1993. 272 p.

SCHERE, R. Concursos 1826-2006. 1. ed. Buenos Aires: Sociedad Central de Arquitectos, 2008. 808 p.

SILVESTRI, G. Ars Pública: ensayos de crítica e historia de la arquitectura, la ciudad y el paisaje. 1. ed. Buenos Aires: SCA/Nobuko, 2011. 348 p.

SOLSONA, J. Solsona: Justo Solsona. Entrevistas. Apuntes para una autobiografía. 1. ed. Buenos Aires: Ediciones Infinito, $1997.182 \mathrm{p}$.

SUÁREZ, O. Concursos: opiniones. In: AAVV. Revista SUMMA N $N^{\circ}$ 50. Buenos Aires: Ediciones Summa SACIFI, 1972. p. 93-100.

WAISMAN, M. Una década revolucionaria: 1960-1970. In: AAVV. Revista SUMMA N ${ }^{\circ}$ 200/201. Buenos Aires, Ediciones Summa SA., 1984. p. 61-62. 